\title{
DERIVATIVE OF A FUNCTION OF A NONSYMMETRIC SECOND-ORDER TENSOR
}

\author{
$\mathrm{BY}$ \\ B. BALENDRAN AND SIA NEMAT-NASSER
}

Department of Applied Mathematics and Engineering Sciences, University of California, San Diego

\begin{abstract}
Exact explicit expressions are obtained for an isotropic tensor-valued function of a nonsymmetric second-order tensor, and its derivative, without resort to eigenvector calculations. These are then used to derive explicit expressions for the material time derivative of the general strain measures in terms of the deformation rate tensor.
\end{abstract}

1. Introduction. Isotropic tensor-valued functions of symmetric second-order tensors are used to express various strain measures in the kinematics of finite deformation. These strain measures are often expressed in a spectral form with respect to the principal triad of the right stretch tensor (left stretch tensor, if the strain measure is Eulerian); Hill [5]. Using the Hamilton-Cayley theorem, a strain measure of this kind can be represented in the coordinate-invariant form. Ting [14] obtains closed-form expressions for the general isotropic functions of a symmetric second-order tensor. Among the various strain measures, logarithmic strain is considered to have certain advantages in constitutive modeling; Hill [6]. However, the relation between the rate of stretch (or deformation rate) and the material time derivative of the logarithmic strain is very complicated. Hill [6] obtains this relation in component form with respect to the principal triad of the right stretch tensor. Later, Hill [7] obtains a relation between the deformation rate and the material time derivative of a general strain measure. This too is in component form with respect to the principal triad of the right stretch tensor. Gurtin and Spear [4] obtain a relation for the Jaumann derivative of the logarithmic strain in terms of the deformation rate and the spin of the principal stretches. Carlson and Hoger [2] obtain a coordinate-invariant expression for the derivative of isotropic tensor-valued functions of a symmetric second-order tensor. Hoger [8] uses this expression to obtain a coordinateinvariant expression for the material time derivative of the logarithmic strain. Scheidler [11] provides an alternative proof for Hill's formula [7] when the principal stretches are repeated. Scheidler [12] then gives approximate coordinate-invariant formulas for the time derivative of the generalized strain tensors.

The isotropic tensor-valued functions of symmetric second-order tensors, and their time derivatives have received considerable attention in the kinematics of finite deformation. Those of nonsymmetric tensors, however, do not seem to have been addressed. In

Received May 2, 1994. 
this paper, exact explicit coordinate-invariant expressions are given for general isotropic tensor-valued functions of nonsymmetric second-order tensors, and their derivatives. Then these results are used to express the material time derivative of the general strain measures in terms of the deformation rate tensor.

As an illustration of the application of the isotropic functions of nonsymmetric secondorder tensors, let the deformation gradients of a material neighborhood at time $t$ and $t+\Delta t$ be related by

$$
\mathbf{F}(t+\Delta t)=\mathbf{F}_{t}(\Delta t) \mathbf{F}(t)
$$

where $\mathbf{F}_{t}(\Delta t)$ is the incremental deformation gradient from time $t$ to $t+\Delta t$. Let $\mathbf{F}_{t}(\Delta t)$ correspond to a constant velocity gradient $\mathbf{L}$ over the time interval $0 \leq \xi \leq \Delta t$. Then,

$$
\mathbf{L}=\dot{\mathbf{F}}_{t}(\xi) \mathbf{F}_{t}^{-1}(\xi), \quad 0 \leq \xi \leq \Delta t
$$

Integration of this rate equation subject to the condition $\mathbf{F}_{t}(0)=\mathbf{1}$ results in

$$
\mathbf{L}=\frac{1}{\Delta t} \ln \left\{\mathbf{F}_{t}(\Delta t)\right\}
$$

In this example the determinant of $\mathbf{F}_{t}$ is positive. However, the results presented here apply to any real-valued second-order tensor. Similarly, if a material element is subjected to constant velocity gradient $\mathbf{L}$ over the time increment $\Delta t$, the incremental deformation gradient becomes

$$
\mathbf{F}_{t}(\Delta t)=\exp (\mathbf{L} \Delta t)
$$

To our knowledge, there is no explicit coordinate-invariant expression for the logarithm or for the exponential of a general real-valued nonsymmetric second-order tensor which may have any set of three eigenvalues, real or complex. In most finite-element codes, for example, (1.3) is generally evaluated approximately.

Results are presented in a three-dimensional setting. When these tensors admit distinct eigenvalues, these results directly carry over to any dimensions. For repeated eigenvalues, the same procedure given for the three dimensions also applies to higher dimensions, but the computations become rather complicated.

2. Spectral representations of nonsymmetric tensors. Let $\mathbf{A}$ be a threedimensional real-valued nonsymmetric second-order tensor with eigenvalues $\lambda_{i}$, and the corresponding eigenvectors $\mathbf{e}_{i}, i=1,2,3$. Its adjoint then is its transpose, $\mathbf{A}^{\mathrm{T}}$. The eigenvalues are the roots of the characteristic equation,

$$
\lambda^{3}-\mathrm{I}_{\mathbf{A}} \lambda^{2}+\mathrm{II}_{\mathbf{A}} \lambda-\mathrm{III}_{\mathbf{A}}=0
$$

where $\mathrm{I}_{\mathbf{A}}, \mathrm{II}_{\mathbf{A}}$, and $\mathrm{III}_{\mathbf{A}}$ are the basic invariants of $\mathbf{A}$, given by

$$
\begin{aligned}
\mathrm{I}_{\mathbf{A}} & =\operatorname{tr}(\mathbf{A}), \\
\mathrm{II}_{\mathbf{A}} & =\frac{1}{2}\left\{\mathrm{I}_{\mathbf{A}}^{2}-\operatorname{tr}\left(\mathbf{A}^{2}\right)\right\}, \\
\mathrm{III}_{\mathbf{A}} & =\frac{1}{3}\left\{\operatorname{tr}\left(\mathbf{A}^{3}\right)-\mathrm{I}_{\mathbf{A}} \operatorname{tr}\left(\mathbf{A}^{2}\right)+\mathrm{II}_{\mathbf{A}} \operatorname{tr}(\mathbf{A})\right\} .
\end{aligned}
$$


The eigenvalues may be real or complex. If $\mathbf{A}$ is symmetric, it is known that the eigenvalues are real, and the eigenvectors are real and orthogonal. However, for a general, real-valued nonsymmetric $\mathbf{A}$, the eigenvectors $\mathbf{e}_{i}, i=1,2,3$, are not orthogonal, and the eigenvalues may not be real. Since the characteristic equation for $\mathbf{A}^{\mathrm{T}}$ is the same as that for $\mathbf{A}$, their eigenvalues are also the same. Denote the eigenvectors of $\mathbf{A}^{\mathrm{T}}$, corresponding to the eigenvalues $\lambda_{i}$, by $\tilde{\mathbf{e}}_{i}, i=1,2,3$. It then follows that ${ }^{1}$

$$
\begin{array}{cl}
\mathbf{A} \mathbf{e}_{i}=\lambda_{i} \mathbf{e}_{i}, & \mathbf{A}^{\mathrm{T}} \tilde{\mathbf{e}}_{j}=\lambda_{j} \tilde{\mathbf{e}}_{j}, \\
\mathbf{e}_{i} \cdot \tilde{\mathbf{e}}_{j}=0 & \text { if } \lambda_{i} \neq \lambda_{j} .
\end{array}
$$

The reciprocal vectors of $\mathbf{A}$ are defined as

$$
\hat{\mathbf{e}}_{i} \equiv \tilde{\mathbf{e}}_{i} /\left(\mathbf{e}_{i} \cdot \tilde{\mathbf{e}}_{i}\right), \quad i=1,2,3
$$

From this and $(2.3 \mathrm{c})$ it follows that

$$
\mathbf{e}_{i} \cdot \hat{\mathbf{e}}_{j}=\delta_{i j}
$$

when the eigenvalues are all distinct. Note that, while the $\mathbf{e}_{i}$ 's may be rendered as unit vectors, in general, $\hat{\mathbf{e}}_{i}$ 's defined by $(2.4 \mathrm{~b})$ are not unit vectors.

When the eigenvalues are distinct, $\mathbf{A}$ can be written in terms of the eigenvalues, eigenvectors, and reciprocal vectors as (spectral representation)

$$
\mathbf{A}=\sum_{i=1}^{3} \lambda_{i} \mathbf{e}_{i} \otimes \hat{\mathbf{e}}_{i}
$$

It follows from (2.3c), (2.4), and (2.5a) that, for any integer $m, \mathbf{A}^{m}$ has the spectral representation,

$$
\mathbf{A}^{m}=\sum_{i=1}^{3} \lambda_{i}^{m} \mathbf{e}_{i} \otimes \hat{\mathbf{e}}_{i}
$$

Now, consider the case where two of the three eigenvalues of $\mathbf{A}$ are equal, say $\lambda_{2}=\lambda_{3}$. Then $\mathbf{e}_{1}$ and $\hat{\mathbf{e}}_{1}$ are uniquely determined. If $\mathbf{A}$ is symmetric, any vector that is orthogonal to $\hat{\mathbf{e}}_{1}=\mathbf{e}_{1}$ is an eigenvector corresponding to $\lambda_{2}=\lambda_{3}$. However, if $\mathbf{A}$ is nonsymmetric, it has only one eigenvector, $\mathbf{e}_{2}$, corresponding to $\lambda_{2}=\lambda_{3}$. Then the reciprocal vector corresponding to this repeated eigenvalue is $\hat{\mathbf{e}}_{3}$, which is orthogonal to $\mathbf{e}_{1}$ and $\mathbf{e}_{2}$, i.e.,

$$
\begin{array}{cc}
\mathbf{A} \mathbf{e}_{1}=\lambda_{1} \mathbf{e}_{1}, & \mathbf{A}^{\mathrm{T}} \hat{\mathbf{e}}_{1}=\lambda_{1} \hat{\mathbf{e}}_{1}, \\
\mathbf{A} \mathbf{e}_{2}=\lambda_{2} \mathbf{e}_{2}, & \mathbf{A}^{\mathrm{T}} \hat{\mathbf{e}}_{3}=\lambda_{2} \hat{\mathbf{e}}_{3}, \\
\mathbf{e}_{2} \cdot \hat{\mathbf{e}}_{1}=\mathbf{e}_{2} \cdot \hat{\mathbf{e}}_{3}=\mathbf{e}_{1} \cdot \hat{\mathbf{e}}_{3}=0 .
\end{array}
$$

Now, any vector other than $\hat{\mathbf{e}}_{3}$, in the plane normal to $\mathbf{e}_{1}$, can be chosen as $\hat{\mathbf{e}}_{2}$. Once $\hat{\mathbf{e}}_{2}$ is chosen, then $\hat{\mathbf{e}}_{3}$, orthogonal to $\hat{\mathbf{e}}_{1}$ and $\hat{\mathbf{e}}_{2}$, is unique. Noting that $\mathbf{e}_{3}$ is not necessarily an eigenvector of $\mathbf{A}$, this tensor can be written as follows:

$$
\mathbf{A}=\sum_{i=1}^{3} \lambda_{i} \mathbf{e}_{i} \otimes \hat{\mathbf{e}}_{i}+\mu_{23} \mathbf{e}_{2} \otimes \hat{\mathbf{e}}_{3}, \quad \mu_{i j}=\hat{\mathbf{e}}_{i} \cdot \mathbf{A} \mathbf{e}_{j}
$$

\footnotetext{
${ }^{1}$ When the eigenvectors $\mathbf{e}_{i}$ and $\tilde{\mathbf{e}}_{j}$ are complex, $\mathbf{e}_{i} \cdot \tilde{\mathbf{e}}_{j}$ is the simple product of $\mathbf{e}_{i}$ and $\tilde{\mathbf{e}}_{j}$, i.e., $\mathbf{e}_{i} \cdot \tilde{\mathbf{e}}_{j}=\left(\mathbf{x}_{i} \cdot \tilde{\mathbf{x}}_{j}-\mathbf{y}_{i} \cdot \tilde{\mathbf{y}}_{j}\right)+i\left(\mathbf{x}_{i} \cdot \tilde{\mathbf{y}}_{j}+\mathbf{y}_{i} \cdot \tilde{\mathbf{x}}_{j}\right)$ for $\mathbf{e}_{i}=\mathbf{x}_{i}+i \mathbf{y}_{i}$ and $\tilde{\mathbf{e}}_{j}=\tilde{\mathbf{x}}_{j}+i \tilde{\mathbf{y}}_{j}$.
} 
Note that, when $\mathbf{e}_{3}$ is an eigenvector, then $\mu_{233}$ is automatically zero. Representation (2.7a), therefore, also applies when there are three distinct eigenvalues.

It follows from (2.7) that

$$
\mathbf{A}^{m}=\sum_{i=1}^{3} \lambda_{i}^{m} \mathbf{e}_{i} \otimes \hat{\mathbf{e}}_{i}+m \lambda_{2}^{m-1} \mu_{23} \mathbf{e}_{2} \otimes \hat{\mathbf{e}}_{3}
$$

Since $\lambda_{1}$ and $\lambda_{2}$ are the roots of

$$
\lambda^{2}-\left(\lambda_{1}+\lambda_{2}\right) \lambda+\lambda_{1} \lambda_{2}=0
$$

it follows from $(2.7)$ that

$$
\mathbf{A}^{2}-\left(\lambda_{1}+\lambda_{2}\right) \mathbf{A}+\lambda_{1} \lambda_{2} \mathbf{1}=\left(\lambda_{2}-\lambda_{1}\right) \mathbf{H}, \quad \mathbf{H}=\mu_{23} \mathbf{e}_{2} \otimes \hat{\mathbf{e}}_{3} .
$$

Note that

$$
\mathbf{H A}=\mathbf{A H}=\lambda_{2} \mathbf{H}, \quad \operatorname{tr}(\mathbf{H})=\operatorname{tr}(\mathbf{A H})=\operatorname{tr}\left(\mathbf{A}^{2} \mathbf{H}\right)=0
$$

Similarly, if all three eigenvalues are identical, say equal to $\lambda$, then $\mathbf{A}$ can be written as

$$
\mathbf{A}=\lambda \mathbf{1}+\mu_{12} \mathbf{e}_{1} \otimes \hat{\mathbf{e}}_{2}+\mu_{13} \mathbf{e}_{1} \otimes \hat{\mathbf{e}}_{3}+\mu_{32} \mathbf{e}_{3} \otimes \hat{\mathbf{e}}_{2}
$$

where $\mu_{i j}$ is defined by $(2.7 \mathrm{~b})$. For this case, it follows that

$$
\begin{aligned}
\mathbf{A}^{m}=\lambda^{m} \mathbf{1} & +\frac{1}{2} m(m-1) \lambda^{m-2} \mu_{13} \mu_{32} \mathbf{e}_{1} \otimes \hat{\mathbf{e}}_{2} \\
& +m \lambda^{m-1}\left(\mu_{12} \mathbf{e}_{1} \otimes \hat{\mathbf{e}}_{2}+\mu_{13} \mathbf{e}_{1} \otimes \hat{\mathbf{e}}_{3}+\mu_{32} \mathbf{e}_{3} \otimes \hat{\mathbf{e}}_{2}\right) .
\end{aligned}
$$

Note that, if $\mathbf{A}$ in (2.10) is symmetric, any vector is an eigenvector. Therefore, $\mu_{12}, \mu_{13}$, and $\mu_{23}$ reduce to zero. However, if $\mathbf{A}$ is nonsymmetric, there will be at most two eigenvectors. If $\mathbf{e}_{1}$ is the unique eigenvector of $\mathbf{A}$, then the unique eigenvector of $\mathbf{A}^{\mathrm{T}}$ is $\hat{\mathbf{e}}_{2}$, which is orthogonal to $\mathbf{e}_{1}$. If $\mathbf{e}_{2}$ is an eigenvector of $\mathbf{A}$, then $\hat{\mathbf{e}}_{1}$ is an eigenvector of $\mathbf{A}^{\prime \Gamma}$. In that case $\mu_{12}=\mu_{32}=0$. If $\mathbf{e}_{1}, \mathbf{e}_{2}$, and $\mathbf{e}_{3}$ are all eigenvectors (this is true only for symmetric $\mathbf{A}$ ), then $\mu_{12}=\mu_{13}=\mu_{32}=0$. It follows from $(2.10)$ that

$$
\begin{gathered}
\mathbf{A}=\lambda \mathbf{1}+\mathbf{H}, \quad \mathbf{H}=\mu_{12} \mathbf{e}_{1} \otimes \hat{\mathbf{e}}_{2}+\mu_{13} \mathbf{e}_{1} \otimes \hat{\mathbf{e}}_{3}+\mu_{32} \mathbf{e}_{3} \otimes \hat{\mathbf{e}}_{2}, \\
\mathbf{H}^{2}=\mu_{13} \mu_{32} \mathbf{e}_{1} \otimes \hat{\mathbf{e}}_{2}, \quad \mathbf{H}^{3}=\mathbf{0}, \\
\mathbf{H A}=\mathbf{A H}=\lambda \mathbf{H}+\mathbf{H}^{2} .
\end{gathered}
$$

This spectral representation can be used to extend many results obtained by various authors $[14,2,8]$ for symmetric second-order tensors to nonsymmetric tensors. 
3. General isotropic tensor-valued functions. Consider a polynomial, tensorvalued function of $\mathbf{A}$,

$$
\mathbf{f}(\mathbf{A})=\sum_{i=0}^{n} k_{i} \mathbf{A}^{i}
$$

where $k_{i}$ and $n$ (integer) are constants. This tensor-valued function is isotropic,

$$
\mathbf{f}\left(\mathbf{R}^{\mathrm{T}} \mathbf{A R}\right)=\mathbf{R}^{\mathrm{T}} \mathbf{f}(\mathbf{A}) \mathbf{R}
$$

for any proper orthogonal (rotation) tensor $\mathbf{R}$. Denote by $f$ the corresponding scalarvalued polynomial,

$$
f(x)=\sum_{i=0}^{n} k_{i} x^{i} .
$$

Consider also cases where $n \rightarrow \infty$, provided that the function $f(x)$ is defined and suitably differentiable.

Substituting the spectral representation of $\mathbf{A}$ from the preceding section into (3.1), we obtain

$$
\begin{aligned}
\mathbf{f}(\mathbf{A}) & =\mathbf{f}(\overline{\mathbf{A}})+\mathbf{f}^{\prime}(\overline{\mathbf{A}}) \mathbf{H}+\frac{1}{2} \mathbf{f}^{\prime \prime}(\overline{\mathbf{A}}) \mathbf{H}^{2}, \\
\mathbf{f}(\overline{\mathbf{A}}) & =\sum_{i=1}^{3} f\left(\lambda_{i}\right) \mathbf{e}_{i} \otimes \hat{\mathbf{e}}_{i}, \\
\mathbf{f}^{\prime}(\overline{\mathbf{A}}) & =\sum_{i=1}^{3} f^{\prime}\left(\lambda_{i}\right) \mathbf{e}_{i} \otimes \hat{\mathbf{e}}_{i}, \\
\mathbf{f}^{\prime \prime}(\overline{\mathbf{A}}) & =\sum_{i=1}^{3} f^{\prime \prime}\left(\lambda_{i}\right) \mathbf{e}_{i} \otimes \hat{\mathbf{e}}_{i}, \quad \overline{\mathbf{A}} \equiv \mathbf{A}-\mathbf{H},
\end{aligned}
$$

$$
\begin{aligned}
& \mathbf{H}=\mathbf{0} \quad \text { if } \lambda_{1} \neq \lambda_{2} \neq \lambda_{3} \neq \lambda_{1}, \\
& \mathbf{H}=\frac{1}{\lambda_{2}-\lambda_{1}}\left\{\mathbf{H}^{2}-\left(\lambda_{1}+\lambda_{2}\right) \mathbf{A}+\lambda_{1} \lambda_{2} \mathbf{1}\right\}, \quad \mathbf{H}^{2}=\mathbf{0} \quad \text { if } \lambda_{1} \neq \lambda_{2}=\lambda_{3}, \\
& \mathbf{H}=\mathbf{A}-\lambda \mathbf{1}, \quad \mathbf{H}^{3}=\mathbf{0} \quad \text { if } \lambda_{1}=\lambda_{2}=\lambda_{3}=\lambda .
\end{aligned}
$$

Repeatedly applying the Hamilton-Cayley theorem, the polynomial tensor-valued function (3.1a), including the considered cases with $n \rightarrow \infty$, can also be represented as

$$
\mathbf{f}(\mathbf{A})=a_{0} \mathbf{1}+a_{1} \mathbf{A}+a_{2} \mathbf{A}^{2}
$$

where $a_{0}, a_{1}$, and $a_{2}$ are functions of the basic invariants $\left(\mathrm{I}_{\mathbf{A}}, \mathrm{II}_{\mathbf{A}}\right.$, and $\left.\mathrm{III}_{\mathbf{A}}\right)$ of $\mathbf{A}$. If the eigenvalues of $\mathbf{A}$ are distinct, substitution of (3.2) into (3.4) results in

$$
f\left(\lambda_{i}\right)=a_{0}+a_{1} \lambda_{i}+a_{2} \lambda_{i}^{2}, \quad i=1,2,3 .
$$

If two of the three eigenvalues are identical, say $\lambda_{2}=\lambda_{3}$, then, from (3.2) and (3.4),

$$
\begin{aligned}
f\left(\lambda_{i}\right) & =a_{0}+a_{1} \lambda_{i}+a_{2} \lambda_{i}^{2}, \quad i=1,2, \\
f^{\prime}\left(\lambda_{2}\right) & =a_{1}+2 a_{2} \lambda_{2} .
\end{aligned}
$$


Similarly, if all three eigenvalues are identical, say equal to $\lambda$, then (3.2) and (3.4) yield

$$
\begin{aligned}
f(\lambda) & =a_{0}+a_{1} \lambda+a_{2} \lambda^{2} \\
f^{\prime}(\lambda) & =a_{1}+2 a_{2} \lambda, \quad f^{\prime \prime}(\lambda)=2 a_{2} .
\end{aligned}
$$

In each case, the corresponding set of three linear equations can be solved to obtain $a_{0}, a_{1}$, and $a_{2}$. However, the solution of (3.6) can also be obtained from the solution of (3.5) by taking a limit as $\lambda_{3}$ goes to $\lambda_{2}$. Similarly, the solution of (3.7) can be obtained from that of (3.6) by taking a limit as $\lambda_{2}$ goes to $\lambda_{1}=\lambda$. Therefore, only (3.5) needs to be solved for the coefficients $a_{0}, a_{1}$, and $a_{2}$. The special cases of repeated eigenvalues are then treated by this limiting process. Note that, when $\mathbf{A}$ is symmetric, $(3.6 \mathrm{~b})$ and $(3.7 \mathrm{~b}, \mathrm{c})$ are immaterial, since $\mathbf{H}=\mathbf{0}$ in such a case. For symmetric $\mathbf{A}$, when the eigenvalues are repeated, $\mathbf{f}(\mathbf{A})$ can be expressed as

$$
\begin{aligned}
& \mathbf{f}(\mathbf{A})=\bar{a}_{0} \mathbf{1}+\bar{a}_{1} \mathbf{A} \quad \text { if } \lambda_{1} \neq \lambda_{2}=\lambda_{3}, \\
& \mathbf{f}(\mathbf{A})=\bar{a}_{0} \mathbf{1} \quad \text { if } \lambda_{1}=\lambda_{2}=\lambda_{3}=\lambda .
\end{aligned}
$$

The coefficients are now obtained uniquely from

$$
\begin{aligned}
f\left(\lambda_{i}\right) & =\bar{a}_{0}+\bar{a}_{1} \lambda_{i}, \quad i=1,2, \quad \text { if } \lambda_{1} \neq \lambda_{2}=\lambda_{3}, \\
f(\lambda) & =\bar{a}_{0} \quad \text { if } \lambda_{1}=\lambda_{2}=\lambda_{3} \equiv \lambda .
\end{aligned}
$$

Expression (3.4) for $\mathbf{f}(\mathbf{A})$ is valid whether or not $\mathbf{A}$ is symmetric. The coefficients change, depending on whether the eigenvalues are real or complex. To find the eigenvalues, rewrite (2.1) in the following equivalent form:

$$
\begin{gathered}
\bar{\lambda}^{3}-3 a \bar{\lambda}-2 b=0, \quad \bar{\lambda}=\lambda-\mathrm{I}_{\mathbf{A}} / 3 \\
a=-\mathrm{II}_{\mathbf{A}^{\prime}} / 3, \quad b=\mathrm{III}_{\mathbf{A}^{\prime}} / 2, \quad \mathbf{A}^{\prime}=\mathbf{A}-\left(\mathrm{I}_{\mathbf{A}} / 3\right) \mathbf{1} .
\end{gathered}
$$

If $b^{2}-a^{3}<0$, all three roots of $(3.10 \mathrm{a})$ are real and distinct. Then, the coefficients are given by

$$
\left[\begin{array}{c}
a_{0} \\
-a_{1} \\
a_{2}
\end{array}\right]=\frac{1}{\Delta}\left[\begin{array}{ccc}
\lambda_{2} \lambda_{3} & \lambda_{3} \lambda_{1} & \lambda_{1} \lambda_{2} \\
\lambda_{2}+\lambda_{3} & \lambda_{3}+\lambda_{1} & \lambda_{1}+\lambda_{2} \\
1 & 1 & 1
\end{array}\right]\left[\begin{array}{l}
f\left(\lambda_{1}\right)\left(\lambda_{2}-\lambda_{3}\right) \\
f\left(\lambda_{2}\right)\left(\lambda_{3}-\lambda_{1}\right) \\
f\left(\lambda_{3}\right)\left(\lambda_{1}-\lambda_{2}\right)
\end{array}\right]
$$

where

$$
\begin{gathered}
\Delta=-\left(\lambda_{1}-\lambda_{2}\right)\left(\lambda_{2}-\lambda_{3}\right)\left(\lambda_{3}-\lambda_{1}\right), \\
\lambda_{n}=\mathrm{I}_{\mathbf{A}} / 3+2 \sqrt{a} \cos \left(\frac{2 n \pi+\phi}{3}\right), \quad \phi=\cos ^{-1}\left(\frac{b}{a \sqrt{a}}\right) .
\end{gathered}
$$

If $b^{2}-a^{3}>0$, only one eigenvalue is real, say, $\lambda_{1}$. Then the complex eigenvalues are given by

$$
\begin{gathered}
\lambda_{2}=\alpha+i \beta, \quad \lambda_{3}=\alpha-i \beta, \\
\alpha=\left(\mathrm{I}_{\mathbf{A}}-\lambda_{1}\right) / 2, \quad \beta=\sqrt{\mathrm{II}_{\mathbf{A}}-\alpha^{2}-2 \alpha \lambda_{1}}, \\
\lambda_{1}=\mathrm{I}_{\mathbf{A}} / 3+\left(b+\sqrt{b^{2}-a^{3}}\right)^{1 / 3}+\left(b-\sqrt{b^{2}-a^{3}}\right)^{1 / 3} .
\end{gathered}
$$


In terms of $\lambda_{1}, \alpha$, and $\beta$, the coefficients are given by

$$
\left[\begin{array}{c}
a_{0} \\
-a_{1} \\
a_{2}
\end{array}\right]=\frac{1}{\Delta}\left[\begin{array}{ccc}
\alpha^{2}+\beta^{2}, & \lambda_{1}\left(\lambda_{1}-2 \alpha\right) & \lambda_{1}\left(\alpha^{2}-\beta^{2}-\alpha \lambda_{1}\right) \\
2 \alpha & -2 \alpha & \alpha^{2}-\beta^{2}-\lambda_{1}^{2} \\
1 & -1 & \alpha-\lambda_{1}
\end{array}\right]\left[\begin{array}{c}
f\left(\lambda_{1}\right) \\
f^{\mathrm{R}} \\
f^{\mathrm{I}} / \beta
\end{array}\right]
$$

where

$$
\begin{gathered}
f^{\mathrm{R}}=\operatorname{Re}\{f(\alpha+i \beta)\}, \quad f^{\mathrm{I}}=\operatorname{Im}\{f(\alpha+i \beta)\}, \\
\Delta=\left(\lambda_{1}-\alpha\right)^{2}+\beta^{2} .
\end{gathered}
$$

For example, if $f(\lambda)=\ln (\lambda)$, then $f^{\mathrm{R}}$ and $f^{\mathrm{I}}$ are given by

$$
f^{\mathrm{R}}=\frac{1}{2} \ln \left(\alpha^{2}+\beta^{2}\right), \quad f^{\mathrm{I}}=\tan ^{-1}(\beta / \alpha) .
$$

Similarly, if $f(\lambda)=e^{\lambda}$, then

$$
f^{\mathrm{R}}=e^{\alpha} \cos \beta, \quad f^{\mathrm{I}}=e^{\alpha} \sin \beta .
$$

Note that, when $\beta$ goes to zero, $f^{\mathrm{I}} / \beta$ goes to $f^{\prime}(\alpha)$.

If $b^{2}=a^{3} \neq 0$, all three roots of (3.10a) are real and, among them, two are identical. Then the coefficients are obtained by taking the limit in (3.11) as $\lambda_{3}$ goes to $\lambda_{2}$, or in (3.13) as $\beta$ goes to zero, arriving at

$$
\begin{aligned}
& a_{0}=\frac{\lambda_{1} f\left(\lambda_{2}\right)-\lambda_{2} f\left(\lambda_{1}\right)}{\lambda_{1}-\lambda_{2}}+a_{2} \lambda_{1} \lambda_{2}, \\
& a_{1}=\frac{f\left(\lambda_{1}\right)-f\left(\lambda_{2}\right)}{\lambda_{1}-\lambda_{2}}-a_{2}\left(\lambda_{1}+\lambda_{2}\right), \\
& a_{2}=\frac{f\left(\lambda_{1}\right)-f\left(\lambda_{2}\right)-\left(\lambda_{1}-\lambda_{2}\right) f^{\prime}\left(\lambda_{2}\right)}{\left(\lambda_{1}-\lambda_{2}\right)^{2}}
\end{aligned}
$$

where

$$
\lambda_{1}=\frac{1}{3} \mathrm{I}_{\mathbf{A}}+2 \sqrt{a}, \quad \lambda_{2}=\frac{1}{3} \mathrm{I}_{\mathbf{A}}-\sqrt{a} .
$$

Note that, when $\mathbf{A}$ is symmetric and $\lambda_{2}=\lambda_{3}$, from (2.8),

$$
\mathbf{A}^{2}-\left(\lambda_{1}+\lambda_{2}\right) \mathbf{A}+\lambda_{1} \lambda_{2} \mathbf{1}=\mathbf{0} .
$$

In this case we substitute (3.17) into (3.4) and in view of (3.19) obtain

$$
\mathbf{f}(\mathbf{A})=\frac{\lambda_{1} f\left(\lambda_{2}\right)-\lambda_{2} f\left(\lambda_{1}\right)}{\lambda_{1}-\lambda_{2}} \mathbf{1}+\frac{f\left(\lambda_{1}\right)-f\left(\lambda_{2}\right)}{\lambda_{1}-\lambda_{2}} \mathbf{A} .
$$

The same result is obtained from $(3.8 \mathrm{a})$ and $(3.9 \mathrm{a})$.

If $b^{2}=a^{3}=0$, all three roots of (3.10a) are real and identical. Then, by taking limits in (3.17) as $\lambda_{2}$ goes to $\lambda_{1}$, obtain

$$
\begin{aligned}
& a_{0}=f(\lambda)-\lambda f^{\prime}(\lambda)+\frac{1}{2} \lambda^{2} f^{\prime \prime}(\lambda), \\
& a_{1}=f^{\prime}(\lambda)-\lambda f^{\prime \prime}(\lambda), \\
& a_{2}=\frac{1}{2} f^{\prime \prime}(\lambda) .
\end{aligned}
$$

Note that, if $\mathbf{A}$ is symmetric and $\lambda_{1}=\lambda_{2}=\lambda_{3} \equiv \lambda$, then $\mathbf{A}=\lambda \mathbf{1}$. In this case, substitution of (3.21) into (3.4) results in

$$
\mathbf{f}(\mathbf{A})=f(\lambda) \mathbf{1}
$$

This equality is the same as the one obtained from (3.8b) and (3.9b). 
4. Derivative of isotropic tensor-valued functions. Now, consider the derivative of the isotropic tensor-valued function $\mathbf{f}(\mathbf{A})$ with respect to a scalar variable $t$, when $\mathbf{A}$ is a differentiable function of $t$. Denote the derivatives of $\mathbf{A}$ and $\mathbf{f}(\mathbf{A})$ with respect to $t$ by $\dot{\mathbf{A}}$ and $\dot{\mathbf{f}}(\mathbf{A})$, respectively. In general, $\dot{\mathbf{A}}$ can be expressed as

$$
\dot{\mathbf{A}}=\sum_{i=1}^{3} \sum_{j=1}^{3}\left(\hat{\mathbf{e}}_{i} \cdot \dot{\mathbf{A}} \mathbf{e}_{j}\right) \mathbf{e}_{i} \otimes \hat{\mathbf{e}}_{j}
$$

This can be split into a part $\stackrel{*}{\mathbf{A}}$, coaxial with $\mathbf{A}$, and a (noncoaxial) remaining part $\mathbf{N}_{\mathbf{A}}$,

$$
\dot{\mathbf{A}}=\stackrel{*}{\mathbf{A}}+\mathbf{N}_{\mathbf{A}}
$$

When $\lambda_{1} \neq \lambda_{2} \neq \lambda_{3} \neq \lambda_{1}$, then

$$
\begin{aligned}
\mathbf{A}^{*} & =\sum_{i=1}^{3}\left(\hat{\mathbf{e}}_{i} \cdot \dot{\mathbf{A}} \mathbf{e}_{i}\right) \mathbf{e}_{i} \otimes \hat{\mathbf{e}}_{i}, \\
\mathbf{N}_{\mathbf{A}} & =\mathbf{A X}-\mathbf{X A} \\
\mathbf{X} & =\sum_{i=1}^{3} \sum_{j=1}^{3} \frac{1-\delta_{i j}}{\lambda_{i}-\lambda_{j}}\left(\hat{\mathbf{e}}_{i} \cdot \dot{\mathbf{A}} \mathbf{e}_{j}\right) \mathbf{e}_{i} \otimes \hat{\mathbf{e}}_{j} .
\end{aligned}
$$

When $\lambda_{1} \neq \lambda_{2}=\lambda_{3}$, then

$$
\begin{aligned}
& \stackrel{*}{\mathbf{A}}=\left(\hat{\mathbf{e}}_{1} \cdot \dot{\mathbf{A}} \mathbf{e}_{1}\right) \mathbf{e}_{1} \otimes \hat{\mathbf{e}}_{1}+\frac{1}{2}\left(\hat{\mathbf{e}}_{2} \cdot \dot{\mathbf{A}} \mathbf{e}_{2}+\hat{\mathbf{e}}_{3} \cdot \dot{\mathbf{A}} \mathbf{e}_{3}\right)\left(\mathbf{e}_{2} \otimes \hat{\mathbf{e}}_{2}+\mathbf{e}_{3} \otimes \hat{\mathbf{e}}_{3}\right) \\
& +\left(\hat{\mathbf{e}}_{2} \cdot \dot{\mathbf{A}} \mathbf{e}_{3}\right) \mathbf{e}_{2} \otimes \hat{\mathbf{e}}_{33} \\
& \mathbf{N}_{\mathbf{A}}=\mathbf{N}_{\overline{\mathbf{A}}}+\mathbf{N}_{\mathbf{H}}, \quad \mathbf{N}_{\overline{\mathbf{A}}}=\overline{\mathbf{A}} \mathbf{X}-\mathbf{X} \overline{\mathbf{A}}, \quad \mathbf{N}_{\mathbf{H}}=\mathbf{H Y}-\mathbf{Y H}, \\
& \mathbf{X}=\frac{1}{\lambda_{1}-\lambda_{2}} \sum_{j=2}^{3}\left\{\left(\hat{\mathbf{e}}_{1} \cdot \dot{\mathbf{A}} \mathbf{e}_{j}\right) \mathbf{e}_{1} \otimes \mathbf{e}_{j}-\left(\hat{\mathbf{e}}_{j} \cdot \dot{\mathbf{A}} \mathbf{e}_{1}\right) \mathbf{e}_{j} \otimes \mathbf{e}_{1}\right\}, \\
& \mathbf{Y}=\frac{1}{2 \mu_{23}}\left(\hat{\mathbf{e}}_{2} \cdot \dot{\mathbf{A}} \mathbf{e}_{2}-\hat{\mathbf{e}}_{33} \cdot \dot{\mathbf{A}} \mathbf{e}_{3}\right) \mathbf{e}_{3} \otimes \hat{\mathbf{e}}_{2} \\
& -\frac{1}{2 \mu_{2: 3}}\left(\hat{\mathbf{e}}_{3} \cdot \dot{\mathbf{A}} \mathbf{e}_{2}\right)\left(\mathbf{e}_{2} \otimes \hat{\mathbf{e}}_{2}-\mathbf{e}_{3} \otimes \hat{\mathbf{e}}_{3}\right),
\end{aligned}
$$

where $\overline{\mathbf{A}}=\mathbf{A}-\mathbf{H}$; see (3.2). When the eigenvalues are distinct, a coordinate-invariant expression for $\mathbf{N}_{\mathbf{A}}$ is obtained by solving (see Appendix A)

$$
\mathbf{A N}_{\mathbf{A}}-\mathbf{N}_{\mathbf{A}} \mathbf{A}=\mathbf{A} \dot{\mathbf{A}}-\dot{\mathbf{A}} \mathbf{A} \quad \text { for } \lambda_{1} \neq \lambda_{2} \neq \lambda_{3} \neq \lambda_{1}
$$

to arrive at

$$
\begin{aligned}
\mathbf{N}_{\mathbf{A}}=\frac{1}{4 \mathrm{II}_{\mathbf{A}^{\prime}}^{3}+27 \mathrm{III}_{\mathbf{A}^{\prime}}^{2}}\left\{18 \mathrm{III}_{\mathbf{A}^{\prime}}^{2} \dot{\mathbf{A}}-6 \mathrm{II}_{\mathbf{A}^{\prime}} \mathrm{III}_{\mathbf{A}^{\prime}}\left(\mathbf{A}^{\prime} \dot{\mathbf{A}}+\dot{\mathbf{A}} \mathbf{A}^{\prime}\right)\right. \\
+2 \mathrm{II}_{\mathbf{A}^{\prime}}^{2} \mathbf{A}^{\prime} \dot{\mathbf{A}} \mathbf{A}^{\prime}-4 \mathrm{II}_{\mathbf{A}^{\prime}}^{2}\left(\mathbf{A}^{\prime 2} \dot{\mathbf{A}}+\dot{\mathbf{A}} \mathbf{A}^{\prime 2}\right) \\
\left.-9 \mathrm{III}_{\mathbf{A}^{\prime}} \mathbf{A}^{\prime}\left(\mathbf{A}^{\prime} \dot{\mathbf{A}}+\dot{\mathbf{A}} \mathbf{A}^{\prime}\right) \mathbf{A}^{\prime}-6 \mathrm{II}_{\mathbf{A}^{\prime}} \mathbf{A}^{\prime 2} \dot{\mathbf{A}} \mathbf{A}^{\prime 2}\right\} \\
\text { for } \lambda_{1} \neq \lambda_{2} \neq \lambda_{3} \neq \lambda_{1} .
\end{aligned}
$$


When $\lambda_{1} \neq \lambda_{2}=\lambda_{3}$, due to the presence of $\mathbf{N}_{\mathbf{H}}$, it is not trivial to find a coordinateinvariant expression for $\mathbf{N}_{\mathbf{A}}$. However, by solving (see Appendix A)

$$
\overline{\mathbf{A}} \mathbf{N}_{\overline{\mathbf{A}}}-\mathbf{N}_{\overline{\mathbf{A}}} \overline{\mathbf{A}}=\overline{\mathbf{A}} \dot{\mathbf{A}}-\dot{\mathbf{A}} \overline{\mathbf{A}}
$$

a coordinate-invariant expression for $\mathbf{N}_{\bar{A}}$ is obtained,

$$
\mathbf{N}_{\overline{\mathbf{A}}}=\frac{1}{\left(\lambda_{1}-\lambda_{2}\right)^{2}}\left\{\left(\lambda_{1}+\lambda_{2}\right)(\overline{\mathbf{A}} \dot{\mathbf{A}}+\dot{\mathbf{A}} \overline{\mathbf{A}})-2 \lambda_{1} \lambda_{2} \dot{\mathbf{A}}-2 \overline{\mathbf{A}} \dot{\mathbf{A}} \overline{\mathbf{A}}\right\}
$$

Differentiate (3.1) with respect to $t$, and substitute from (4.1) to obtain

$$
\begin{aligned}
\dot{\mathbf{f}}(\mathbf{A})=\mathbf{f}^{\prime}(\mathbf{A}) \stackrel{*}{\mathbf{A}}+ & \mathbf{N}_{\mathbf{f}} \quad \text { for } \lambda_{1} \neq \lambda_{2} \neq \lambda_{3} \neq \lambda_{1}, \\
\dot{\mathbf{f}}(\mathbf{A})=\mathbf{f}^{\prime}(\mathbf{A}) \stackrel{*}{\mathbf{A}} & +\frac{1}{2} f^{\prime \prime}\left(\lambda_{2}\right) \operatorname{tr}(\dot{\mathbf{A}} \mathbf{H})\left(\mathbf{e}_{2} \otimes \hat{\mathbf{e}}_{2}+\mathbf{e}_{3} \otimes \hat{\mathbf{e}}_{3}\right) \\
& +\frac{1}{6} f^{\prime \prime \prime}\left(\lambda_{2}\right) \operatorname{tr}(\dot{\mathbf{A}} \mathbf{H}) \mathbf{H}+\mathbf{N}_{\mathbf{f}} \quad \text { for } \lambda_{1} \neq \lambda_{2}=\lambda_{3}, \\
\dot{\mathbf{f}}(\mathbf{A})=f^{\prime}(\lambda) \dot{\mathbf{A}} & +\frac{1}{2} f^{\prime \prime}(\lambda)(\mathbf{H} \dot{\mathbf{A}}+\dot{\mathbf{A}} \mathbf{H}) \\
& +\frac{1}{6} f^{\prime \prime \prime}(\lambda)\left(\mathbf{H}^{2} \dot{\mathbf{A}}+\dot{\mathbf{A}} \mathbf{H}^{2}+\mathbf{H} \dot{\mathbf{A}} \mathbf{H}\right) \\
& +\frac{1}{24} f^{\prime \prime \prime \prime}(\lambda)\left(\mathbf{H} \dot{\mathbf{A}} \mathbf{H}^{2}+\mathbf{H}^{2} \dot{\mathbf{A}} \mathbf{H}\right) \\
& +\frac{1}{120} f^{\prime \prime \prime \prime \prime}(\lambda) \mathbf{H}^{2} \dot{\mathbf{A}} \mathbf{H}^{2} \quad \text { for } \lambda_{1}=\lambda_{2}=\lambda_{3}=\lambda,
\end{aligned}
$$

where the tensor $\mathbf{f}^{\prime}(\mathbf{A})$, coaxial with $\mathbf{A}$, is given by

$$
\mathbf{f}^{\prime}(\mathbf{A})=\sum_{i=0}^{n} i k_{i} \mathbf{A}^{i-1}
$$

and $\mathbf{N}_{\mathbf{f}}$ is the noncoaxial part of $\dot{\mathbf{f}}(\mathbf{A})$ such that

$$
\begin{aligned}
& \hat{\mathbf{e}}_{i} \cdot \mathbf{N}_{\mathbf{f}} \mathbf{e}_{i}=0, \quad i=1,2,3, \quad \text { for } \lambda_{1} \neq \lambda_{2} \neq \lambda_{3} \neq \lambda_{1}, \\
& \hat{\mathbf{e}}_{1} \cdot \mathbf{N}_{\mathbf{f}} \mathbf{e}_{1}=\hat{\mathbf{e}}_{2} \cdot \mathbf{N}_{\mathbf{f}} \mathbf{e}_{3}=\hat{\mathbf{e}}_{2} \cdot \mathbf{N}_{\mathbf{f}} \mathbf{e}_{2}+\hat{\mathbf{e}}_{3} \cdot \mathbf{N}_{\mathbf{f}} \mathbf{e}_{3}=0 \text { for } \lambda_{1} \neq \lambda_{2}=\lambda_{3} .
\end{aligned}
$$

In general, $\mathbf{f}^{\prime}(\mathbf{A})$ can be expressed as

$$
\mathbf{f}^{\prime}(\mathbf{A})=b_{0} \mathbf{1}+b_{1} \mathbf{A}+b_{2} \mathbf{A}^{2}
$$

where the coefficients $b_{0}, b_{1}$, and $b_{2}$ are functions of the eigenvalues of $\mathbf{A}$ and can be obtained by replacing $f(\lambda)$ by $f^{\prime}(\lambda)$ in the relevant expressions developed in the preceding section. However, a closed-form coordinate-invariant expression for the noncoaxial part, $\mathbf{N}_{\mathbf{f}}$, is not produced through differentiation of (3.1). Hence, consider (3.4).

Differentiation of (3.4) results in

$$
\dot{\mathbf{f}}(\mathbf{A})=a_{1} \dot{\mathbf{A}}+a_{2}(\mathbf{A} \dot{\mathbf{A}}+\dot{\mathbf{A}} \mathbf{A})+c_{0} \mathbf{1}+c_{1} \mathbf{A}+c_{2} \mathbf{A}^{2},
$$


where the unknown coefficients $c_{0}, c_{1}$, and $c_{2}$ are functions of $\mathbf{A}$ and $\dot{\mathbf{A}}$. Comparing the noncoaxial terms in (4.6) and (4.8), we obtain the coordinate-invariant expression for $\mathbf{N}_{\mathbf{f}}$ in closed form as

$$
\begin{gathered}
\mathbf{N}_{\mathbf{f}}=a_{1}\left(\dot{\mathbf{A}}-\mathbf{A}^{*}\right)+a_{2}(\mathbf{A} \dot{\mathbf{A}}+\dot{\mathbf{A}} \mathbf{A}-2 \mathbf{A} \mathbf{A}) \quad \text { for } \lambda_{1} \neq \lambda_{2} \neq \lambda_{3} \neq \lambda_{1}, \\
\mathbf{N}_{\mathbf{f}}=a_{1}(\dot{\mathbf{A}}-\mathbf{A})+a_{2}(\mathbf{A} \dot{\mathbf{A}}+\dot{\mathbf{A}} \mathbf{A}-2 \mathbf{A} \mathbf{A})-a_{2} \operatorname{tr}(\dot{\mathbf{A}} \mathbf{H})\left(\mathbf{e}_{2} \otimes \hat{\mathbf{e}}_{2}+\mathbf{e}_{3} \otimes \hat{\mathbf{e}}_{3}\right) \\
\text { for } \lambda_{1} \neq \lambda_{2}=\lambda_{3} .
\end{gathered}
$$

Substitution of (4.9) into (4.6) results in

$$
\begin{aligned}
& \dot{\mathbf{f}}(\mathbf{A})=a_{1} \dot{\mathbf{A}}+a_{2}(\mathbf{A} \dot{\mathbf{A}}+\dot{\mathbf{A}} \mathbf{A})+\left\{\mathbf{f}^{\prime}(\mathbf{A})-a_{1} \mathbf{1}-2 a_{2} \mathbf{A}\right\} \stackrel{*}{\mathbf{A}}, \\
& \text { for } \lambda_{1} \neq \lambda_{2} \neq \lambda_{3} \neq \lambda_{1}, \\
& \dot{\mathbf{f}}(\mathbf{A})=a_{1} \dot{\mathbf{A}}+a_{2}(\mathbf{A} \dot{\mathbf{A}}+\dot{\mathbf{A}} \mathbf{A})+\left\{\mathbf{f}^{\prime}(\mathbf{A})-a_{1} \mathbf{1}-2 a_{2} \mathbf{A}\right\} \stackrel{*}{\mathbf{A}} \\
&+\frac{1}{2}\left\{f^{\prime \prime}\left(\lambda_{2}\right)-2 a_{2}\right\} \operatorname{tr}(\dot{\mathbf{A}} \mathbf{H})\left(\mathbf{e}_{2} \otimes \hat{\mathbf{e}}_{2}+\mathbf{e}_{3} \otimes \hat{\mathbf{e}}_{3}\right) \\
&+\frac{1}{6} f^{\prime \prime \prime}\left(\lambda_{2}\right) \operatorname{tr}(\dot{\mathbf{A}} \mathbf{H}) \mathbf{H} \quad \text { for } \lambda_{1} \neq \lambda_{2}=\lambda_{3} .
\end{aligned}
$$

Therefore, to complete the derivation of the derivative of $\mathbf{f}(\mathbf{A})$, it is only necessary to find a coordinate-invariant expression for $\mathbf{A}^{*}$ in terms of $\mathbf{A}$ and $\dot{\mathbf{A}}$. When all three eigenvalues are distinct, a simple method is to note that $\mathbf{A}^{*}$ is coaxial with $\mathbf{A}$, and write

$$
\stackrel{*}{\mathbf{A}}=\sum_{i=1}^{3} \operatorname{tr}\left(\dot{\mathbf{A}} \mathbf{E}_{i}\right) \mathbf{E}_{i}, \quad \mathbf{E}_{i} \equiv \mathbf{e}_{i} \otimes \hat{\mathbf{e}}_{i} \quad \text { for } \lambda_{1} \neq \lambda_{2} \neq \lambda_{3} \neq \lambda_{1}
$$

Observe that, since $\mathbf{E}_{i}, i=1,2,3$, is coaxial with $\mathbf{A}$, it can be written as

$$
\mathbf{E}_{i}=\alpha_{0}^{i} \mathbf{1}+\alpha_{1}^{i} \mathbf{A}+\alpha_{2}^{i} \mathbf{A}^{2}
$$

where the coefficients $\alpha_{0}^{i}, \alpha_{1}^{i}$, and $\alpha_{2}^{i}$ are obtained from

$$
\alpha_{0}^{i}+\alpha_{i}^{i} \lambda_{j}+\alpha_{2}^{i} \lambda_{j}^{2}=\delta_{i j}, \quad j=1,2,3
$$

This yields the following coordinate-invariant expression for $\mathbf{E}_{i}$ :

$$
\mathbf{E}_{i}=\frac{1}{\left(\lambda_{i}-\lambda_{j}\right)\left(\lambda_{i}-\lambda_{k}\right)}\left\{\mathbf{A}^{2}-\left(\lambda_{j}+\lambda_{k}\right) \mathbf{A}+\lambda_{j} \lambda_{k} \mathbf{1}\right\}
$$

where $i, j$, and $k$ are permutations of 1,2 , and 3. Substitution of (4.11a) into (4.10) results in

$$
\begin{aligned}
\dot{\mathbf{f}}(\mathbf{A})=a_{1} \dot{\mathbf{A}}+a_{2}(\mathbf{A} \dot{\mathbf{A}}+\dot{\mathbf{A}} \mathbf{A})+\sum_{i=1}^{3}\left\{f^{\prime}\left(\lambda_{i}\right)-a_{1}-2 a_{2} \lambda_{i}\right\} & \operatorname{tr}\left(\dot{\mathbf{A}} \mathbf{E}_{i}\right) \mathbf{E}_{i}, \\
& \text { for } \lambda_{1} \neq \lambda_{2} \neq \lambda_{3} \neq \lambda_{1} .
\end{aligned}
$$


When any two of the three eigenvalues are equal, say $\lambda_{1} \neq \lambda_{2}=\lambda_{3}$, the tensors coaxial with $\mathbf{A}$ can be expressed with respect to the orthonormal bases $\left\{\mathbf{E}_{1},\left(\mathbf{1}-\mathbf{E}_{1}\right), \mathbf{H}\right\}$ as follows:

$$
\begin{gathered}
\mathbf{A}=\lambda_{1} \mathbf{E}_{1}+\lambda_{2}\left(\mathbf{1}-\mathbf{E}_{1}\right)+\mathbf{H} \\
\mathbf{f}^{\prime}(\mathbf{A})=f^{\prime}\left(\lambda_{1}\right) \mathbf{E}_{1}+f^{\prime}\left(\lambda_{2}\right)\left(\mathbf{1}-\mathbf{E}_{1}\right)+f^{\prime \prime}\left(\lambda_{2}\right) \mathbf{H}, \\
\mathbf{A}=\operatorname{tr}\left(\dot{\mathbf{A}} \mathbf{E}_{1}\right) \mathbf{E}_{1}+\frac{1}{2}\left\{\operatorname{tr}(\dot{\mathbf{A}})-\operatorname{tr}\left(\dot{\mathbf{A}} \mathbf{E}_{1}\right)\right\}\left(\mathbf{1}-\mathbf{E}_{1}\right)+\alpha_{1} \mathbf{H}, \\
\mathbf{E}_{1} \equiv \mathbf{e}_{1} \otimes \hat{\mathbf{e}}_{1}=\frac{1}{\lambda_{1}-\lambda_{2}}\left(\overline{\mathbf{A}}-\lambda_{2} \mathbf{1}\right), \quad \overline{\mathbf{A}}=\mathbf{A}-\mathbf{H}, \\
\mathbf{H}=\frac{1}{\lambda_{2}-\lambda_{1}}\left\{\mathbf{A}^{2}-\left(\lambda_{1}+\lambda_{2}\right) \mathbf{A}+\lambda_{1} \lambda_{2} \mathbf{1}\right\},
\end{gathered}
$$

where the coefficient $\alpha_{1}$ is still unknown. However, it is not necessary to evaluate $\alpha_{1}$, in order to obtain an explicit expression for $\dot{\mathbf{f}}(\mathbf{A})$. Substituting (4.13) into (4.10) in view of (3.17), and noting that $\mathbf{E}_{1} \mathbf{H}=\mathbf{H}^{2}=\mathbf{0}$, we obtain

$$
\begin{aligned}
\dot{\mathbf{f}}(\mathbf{A})=a_{1} \dot{\mathbf{A}} & +a_{2}(\mathbf{A} \dot{\mathbf{A}}+\dot{\mathbf{A}} \mathbf{A})+\left\{f^{\prime}\left(\lambda_{1}\right)-a_{1}-2 a_{2} \lambda_{1}\right\} \operatorname{tr}\left(\dot{\mathbf{A}} \mathbf{E}_{1}\right) \mathbf{E}_{1} \\
& +\frac{1}{2}\left\{f^{\prime \prime}\left(\lambda_{2}\right)-2 a_{2}\right\}\left\{\operatorname{tr}(\dot{\mathbf{A}})-\operatorname{tr}\left(\dot{\mathbf{A}} \mathbf{E}_{1}\right)\right\} \mathbf{H} \\
& +\frac{1}{2}\left\{f^{\prime \prime}\left(\lambda_{2}\right)-2 a_{2}\right\} \operatorname{tr}(\dot{\mathbf{A}} \mathbf{H})\left(\mathbf{1}-\mathbf{E}_{1}\right) \\
& +\frac{1}{6} f^{\prime \prime \prime}\left(\lambda_{2}\right) \operatorname{tr}(\dot{\mathbf{A}} \mathbf{H}) \mathbf{H} \quad \text { for } \lambda_{1} \neq \lambda_{2}=\lambda_{3} .
\end{aligned}
$$

The same result is obtained by taking limits as $\lambda_{3}$ goes to $\lambda_{2}$, in (4.12a). Note that, when $\lambda_{3}$ goes to $\lambda_{2}$,

$$
\left(\mathbf{E}_{2}-\mathbf{E}_{3}\right) / 2 \rightarrow\left(\lambda_{2}-\lambda_{3}\right) \mathbf{H}, \quad\left(\mathbf{E}_{2}+\mathbf{E}_{3}\right) \rightarrow\left(\mathbf{1}-\mathbf{E}_{1}\right) \quad \text { as } \lambda_{3} \rightarrow \lambda_{2}
$$

Using Rivlin's identities [10, Eqs. 4.22 and 4.25] in (4.6c) and noting that all three invariants of $\mathbf{H}$ are zero, we arrive at

$$
\begin{aligned}
\dot{\mathbf{f}}(\mathbf{A})=f^{\prime}(\lambda) \dot{\mathbf{A}} & +\frac{1}{2} f^{\prime \prime}(\lambda)(\mathbf{H} \dot{\mathbf{A}}+\dot{\mathbf{A}} \mathbf{H}) \\
& +\frac{1}{6} f^{\prime \prime \prime}(\lambda)\left\{\operatorname{tr}\left(\dot{\mathbf{A}} \mathbf{H}^{2}\right) \mathbf{1}+\operatorname{tr}(\dot{\mathbf{A}} \mathbf{H}) \mathbf{H}+\operatorname{tr}(\dot{\mathbf{A}}) \mathbf{H}^{2}\right\} \\
& +\frac{1}{24} f^{\prime \prime \prime \prime}(\lambda)\left\{\operatorname{tr}\left(\dot{\mathbf{A}} \mathbf{H}^{2}\right) \mathbf{H}+\operatorname{tr}(\dot{\mathbf{A}} \mathbf{H}) \mathbf{H}^{2}\right\} \\
& +\frac{1}{120} f^{\prime \prime \prime \prime \prime}(\lambda) \operatorname{tr}\left(\dot{\mathbf{A}} \mathbf{H}^{2}\right) \mathbf{H}^{2} \quad \text { for } \lambda_{1}=\lambda_{2}=\lambda_{3}=\lambda .
\end{aligned}
$$

The same result is obtained by taking limits as $\lambda_{2}$ goes to $\lambda_{1}=\lambda$ in $(4.12 \mathrm{~b})$. Note the following limits in (4.12b) when $\delta \lambda \equiv \lambda_{1}-\lambda_{2}$ goes to zero:

$$
\begin{gathered}
\delta \lambda \mathbf{E}_{1}+\mathbf{H} \rightarrow \mathbf{A}-\lambda \mathbf{1}, \quad \delta \lambda \mathbf{E}_{1}-\mathbf{H} \rightarrow \frac{2}{\delta \lambda}(\mathbf{A}-\lambda \mathbf{1})^{2}, \\
\frac{1}{\delta \lambda^{2}}\left\{f^{\prime}\left(\lambda_{1}\right)-a_{1}-2 a_{2} \lambda_{1}\right\} \rightarrow \frac{1}{6} f^{\prime \prime \prime}(\lambda)+\frac{\delta \lambda}{12} f^{\prime \prime \prime \prime}(\lambda)+\frac{\delta \lambda^{2}}{40} f^{\prime \prime \prime \prime \prime}(\lambda), \\
\frac{1}{\delta \lambda} f^{\prime \prime}\left(\lambda_{2}\right)-2 a_{2} \rightarrow-\frac{1}{3} f^{\prime \prime \prime}(\lambda)-\frac{\delta \lambda}{12} f^{\prime \prime \prime \prime}(\lambda)-\frac{\delta \lambda^{2}}{70} f^{\prime \prime \prime \prime}(\lambda) \quad \text { as } \delta \lambda \rightarrow 0 .
\end{gathered}
$$


Alternatively, by substituting (4.1) and (4.7b) into (4.10), $\dot{\mathbf{f}}(\mathbf{A})$ is expressed in terms of the noncoaxial part of $\dot{\mathbf{A}}$ as follows:

$$
\begin{aligned}
\dot{\mathbf{f}}(\mathbf{A})=a_{1} \mathbf{N}_{\mathbf{A}} & +a_{2}\left(\mathbf{A} \mathbf{N}_{\mathbf{A}}+\mathbf{N}_{\mathbf{A}} \mathbf{A}\right)+b_{0}\left(\dot{\mathbf{A}}-\mathbf{N}_{\mathbf{A}}\right) \\
& +\frac{1}{2} b_{1}\left\{\mathbf{A}\left(\dot{\mathbf{A}}-\mathbf{N}_{\mathbf{A}}\right)+\left(\dot{\mathbf{A}}-\mathbf{N}_{\mathbf{A}}\right) \mathbf{A}\right\} \\
& +\frac{1}{2} b_{2}\left\{\mathbf{A}^{2}\left(\dot{\mathbf{A}}-\mathbf{N}_{\mathbf{A}}\right)+\left(\dot{\mathbf{A}}-\mathbf{N}_{\mathbf{A}}\right) \mathbf{A}^{2}\right\} \quad \text { for } \lambda_{1} \neq \lambda_{2} \neq \lambda_{3} \neq \lambda_{1} \\
\dot{\mathbf{f}}(\mathbf{A})=a_{1} \mathbf{N}_{\overline{\mathbf{A}}} & +a_{2}\left(\mathbf{A} \mathbf{N}_{\overline{\mathbf{A}}}+\mathbf{N}_{\overline{\mathbf{A}}} \mathbf{A}\right)+b_{0}\left(\dot{\mathbf{A}}-\mathbf{N}_{\overline{\mathbf{A}}}\right) \\
& +\frac{1}{2} b_{1}\left\{\mathbf{A}\left(\dot{\mathbf{A}}-\mathbf{N}_{\overline{\mathbf{A}}}\right)+\left(\dot{\mathbf{A}}-\mathbf{N}_{\overline{\mathbf{A}}}\right) \mathbf{A}\right\} \\
& +\frac{1}{2} b_{2}\left\{\mathbf{A}^{2}\left(\dot{\mathbf{A}}-\mathbf{N}_{\overline{\mathbf{A}}}\right)+\left(\dot{\mathbf{A}}-\mathbf{N}_{\overline{\mathbf{A}}}\right) \mathbf{A}^{2}\right\} \\
& +\frac{1}{2}\left\{f^{\prime \prime}\left(\lambda_{2}\right)-2 a_{2}\right\} \operatorname{tr}(\dot{\mathbf{A}} \mathbf{H})\left(\mathbf{1}-\mathbf{E}_{1}\right) \\
& +\frac{1}{6} f^{\prime \prime \prime}\left(\lambda_{2}\right) \operatorname{tr}(\dot{\mathbf{A}} \mathbf{H}) \mathbf{H} \quad \text { for } \lambda_{1} \neq \lambda_{2}=\lambda_{3} .
\end{aligned}
$$

Therefore, alternate expressions for $\dot{\mathbf{f}}(\mathbf{A})$ are obtained by substituting (4.5) into (4.15). However, these expressions are more complicated than (4.12). It is worth noting that the expressions that result from substitution of (4.5b) into (4.15b) when $\mathbf{A}$ and $\dot{\mathbf{A}}$ are symmetric, are the same as those obtained by Carlson and Hoger [2]. When the eigenvalues are distinct, it follows from (4.11b) that

$$
\mathbf{E}_{i} \dot{\mathbf{A}} \mathbf{E}_{i}=\operatorname{tr}\left(\dot{\mathbf{A}} \mathbf{E}_{i}\right) \mathbf{E}_{i}
$$

Substitution of (4.16) and (4.11c) into (4.12a) results in another alternate expression for $\dot{\mathbf{f}}(\mathbf{A})$. This is the same expression obtained by Carlson and Hoger [2] for symmetric $\mathbf{A}$. When the eigenvalues $\lambda_{2}$ and $\lambda_{3}$ are complex, say,

$$
\lambda_{2}=\alpha+i \beta, \quad \lambda_{3}=\alpha-i \beta,
$$

(4.12a) can be rewritten as follows:

$$
\begin{aligned}
\dot{\mathbf{f}}(\mathbf{A})=a_{1} \dot{\mathbf{A}} & +a_{2}(\mathbf{A} \dot{\mathbf{A}}+\dot{\mathbf{A}} \mathbf{A})+\left\{f^{\prime}\left(\lambda_{1}\right)-a_{1}-2 a_{2} \lambda_{1}\right\} \operatorname{tr}\left(\dot{\mathbf{A}} \mathbf{E}_{1}\right) \mathbf{E}_{1} \\
& +\frac{1}{2}\left(f^{\prime \mathrm{R}}-a_{1}-2 a_{2} \alpha\right)\left\{\operatorname{tr}\left(\dot{\mathbf{A}} \mathbf{E}^{\mathrm{R}}\right) \mathbf{E}^{\mathrm{R}}-\operatorname{tr}\left(\dot{\mathbf{A}} \mathbf{E}^{\mathrm{I}}\right) \mathbf{E}^{\mathrm{I}}\right\} \\
& -\frac{1}{2}\left(f^{\prime \mathrm{I}}-2 a_{2} \beta\right)\left\{\operatorname{tr}\left(\dot{\mathbf{A}} \mathbf{E}^{\mathrm{I}}\right) \mathbf{E}^{\mathrm{R}}+\operatorname{tr}\left(\dot{\mathbf{A}} \mathbf{E}^{\mathrm{R}}\right) \mathbf{E}^{\mathrm{I}}\right\}
\end{aligned}
$$

where

$$
\begin{aligned}
\mathbf{E}_{1} & =\frac{1}{\left(\lambda_{1}-\alpha\right)^{2}+\beta^{2}}\left\{\mathbf{A}^{2}-2 \alpha \mathbf{A}+\left(\alpha^{2}+\beta^{2}\right) \mathbf{1}\right\}, \\
\mathbf{E}^{\mathrm{R}} & =\frac{-1}{\left(\lambda_{1}-\alpha\right)^{2}+\beta^{2}}\left\{\mathbf{A}^{2}-2 \alpha \mathbf{A}-\lambda_{1}\left(\lambda_{1}-2 \alpha\right) \mathbf{1}\right\}, \\
\mathbf{E}^{\mathrm{I}} & =\frac{1}{\beta\left\{\left(\lambda_{1}-\alpha\right)^{2}+\beta^{2}\right\}}\left\{\left(\lambda_{1}-\alpha\right) \mathbf{A}^{2}+\left(\alpha^{2}-\beta^{2}-\lambda_{1}^{2}\right) \mathbf{A}-\lambda_{1}\left(\alpha^{2}-\beta^{2}-\alpha \lambda_{1}\right) \mathbf{1}\right\}, \\
f^{\prime R} & =\operatorname{Re}\left\{f^{\prime}(\alpha+i \beta)\right\}, \quad f^{\prime I}=\operatorname{Im}\left\{f^{\prime}(\alpha+i \beta)\right\} .
\end{aligned}
$$


Observe the following limits when $\beta$ goes to zero:

$$
\begin{aligned}
& \mathbf{E}_{1} \rightarrow \frac{1}{\lambda_{1}-\alpha}(\overline{\mathbf{A}}-\alpha \mathbf{1}), \quad \mathbf{E}^{\mathrm{R}} \rightarrow \mathbf{1}-\mathbf{E}_{1}, \quad \mathbf{E}^{\mathrm{I}} \rightarrow \frac{-1}{\beta} \mathbf{H}, \\
& f^{\prime \mathrm{I}} \rightarrow \beta f^{\prime \prime}(\alpha), \quad f^{\prime \mathrm{R}}-a_{1}-2 a_{2} \alpha \rightarrow \frac{-\beta^{2}}{3} f^{\prime \prime \prime}(\alpha) \quad \text { as } \beta \rightarrow 0,
\end{aligned}
$$

where

$$
\overline{\mathbf{A}}=\mathbf{A}-\mathbf{H}, \quad \mathbf{H}=\frac{1}{\alpha-\lambda_{1}}\left\{\mathbf{A}^{2}-\left(\lambda_{1}+\alpha\right) \mathbf{A}+\alpha \lambda_{1} \mathbf{1}\right\}
$$

This shows that $(4.12 \mathrm{~d})$ reduces to $(4.12 \mathrm{~b})$ when the eigenvalues are real and equal.

5. Strain rates. The general material strain measure, $\mathbf{E}$, in the kinematics of finite deformation is expressed as an isotropic tensor-valued function of the (symmetric) right stretch tensor $\mathbf{U}$,

$$
\mathbf{E}=\mathbf{f}(\mathbf{U})
$$

where $\mathbf{f}$ is in the class of functions considered in Sec. 3. The material time derivative of this strain measure can be related to the stretch tensor $\mathbf{U}$ and its rate $\dot{\mathbf{U}}$, using the results obtained in the preceding section. The deformation rate, $\mathbf{D}$, is related to the stretch rate by

$$
\mathbf{D}=\frac{1}{2} \mathbf{R}\left(\dot{\mathbf{U}} \mathbf{U}^{-1}+\mathbf{U}^{-1} \dot{\mathbf{U}}\right) \mathbf{R}^{\mathrm{T}},
$$

where $\mathbf{R}$ is the rotation tensor, defined through the polar decomposition of the deformation gradient, $\mathbf{f}=\mathbf{R U}$. Therefore, the stretch rate $\dot{\mathbf{U}}$ can be expressed in terms of $\widehat{\mathbf{D}}$ defined by

$$
\widehat{\mathbf{D}}=\mathbf{R}^{\mathrm{T}} \mathbf{D R}
$$

without resorting to eigenvalue calculations; see Hoger [8], Mehrabadi and Nemat-Nasser [9], and Scheidler [13]. Here we seek to obtain a simple coordinate-invariant expression for $\dot{\mathbf{U}}$ in terms of $\widehat{\mathbf{D}}$. This may involve the eigenvalues of $\mathbf{U}$, but requires no eigenvector calculations.

Denote the eigenvalues of $\mathbf{U}$ by $\lambda_{i}, i=1,2,3$. The deformation rate $\widehat{\mathbf{D}}$ is decomposed

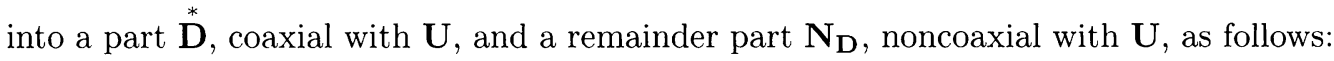

$$
\begin{aligned}
& \widehat{\mathbf{D}}=\stackrel{*}{\mathbf{D}}+\mathbf{N}_{\mathbf{D}}, \\
& \stackrel{*}{\mathbf{D}}=\sum_{i=1}^{3} \operatorname{tr}\left(\widehat{\mathbf{D}} \mathbf{E}_{i}\right) \mathbf{E}_{i} \quad \text { for } \lambda_{1} \neq \lambda_{2} \neq \lambda_{3} \neq \lambda_{1} . \\
& \stackrel{*}{\mathbf{D}}=\operatorname{tr}\left(\widehat{\mathbf{D}} \mathbf{E}_{1}\right) \mathbf{E}_{1}+\frac{1}{2}\left\{\operatorname{tr}(\widehat{\mathbf{D}})-\operatorname{tr}\left(\widehat{\mathbf{D}} \mathbf{E}_{1}\right)\right\}\left(\mathbf{1}-\mathbf{E}_{1}\right) \quad \text { for } \lambda_{1} \neq \lambda_{2}=\lambda_{3}, \\
& \stackrel{*}{\mathbf{D}}=\operatorname{tr}(\widehat{\mathbf{D}}) \mathbf{1} \quad \text { for } \lambda_{1}=\lambda_{2}=\lambda_{3} \equiv \lambda,
\end{aligned}
$$

where $\mathbf{E}_{i}, i=1,2,3$, are defined below by (5.8). Denote by $\dot{\mathbf{U}}$ and $\mathbf{N}_{\mathbf{U}}$ the coaxial and the noncoaxial parts of $\dot{\mathbf{U}}$, respectively. Then, from (5.2), it follows that

$$
\begin{aligned}
\stackrel{*}{\mathbf{U}}=\mathbf{U} & \stackrel{*}{\mathbf{D}}=\stackrel{*}{\mathbf{D}} \mathbf{U} \\
\mathbf{U N}_{\mathbf{U}}+\mathbf{N}_{\mathbf{U}} \mathbf{U} & =2 \mathbf{U} \mathbf{N}_{\mathbf{D}} \mathbf{U}
\end{aligned}
$$


Following Scheidler [13], (5.4b) is solved to obtain

$$
\mathbf{N}_{\mathbf{U}}=\frac{2}{\mathrm{I}_{\mathbf{U}} \mathrm{II}_{\mathbf{U}}-\mathrm{III}_{\mathbf{U}}}\left\{\mathrm{I}_{\mathbf{U}} \mathrm{III}_{\mathbf{U}} \mathbf{N}_{\mathbf{D}}+\mathrm{II}_{\mathbf{U}} \mathbf{U N}_{\mathbf{D}} \mathbf{U}-\mathrm{III}_{\mathbf{U}}\left(\mathbf{U N}_{\mathbf{D}}+\mathbf{N}_{\mathbf{D}} \mathbf{U}\right)\right\}
$$

From (5.3) and (5.4), an expression for the stretch rate $\dot{\mathbf{U}}$ is obtained in terms of $\widehat{\mathbf{D}}$ as follows:

$$
\begin{aligned}
\dot{\mathbf{U}} & =\mathbf{K}_{\mathbf{U}}+\sum_{i=1}^{3}\left\{\lambda_{i} \operatorname{tr}\left(\widehat{\mathbf{D}} \mathbf{E}_{i}\right)-\operatorname{tr}\left(\mathbf{K}_{\mathbf{U}} \mathbf{E}_{i}\right)\right\} \mathbf{E}_{i} \quad \text { for } \lambda_{1} \neq \lambda_{2} \neq \lambda_{3} \neq \lambda_{1}, \\
\dot{\mathbf{U}} & =\mathbf{K}_{\mathbf{U}}+\left\{\lambda_{1} \operatorname{tr}\left(\widehat{\mathbf{D}} \mathbf{E}_{1}\right)-\operatorname{tr}\left(\mathbf{K}_{\mathbf{U}} \mathbf{E}_{1}\right)\right\} \mathbf{E}_{1} \quad \text { for } \lambda_{1} \neq \lambda_{2}=\lambda_{3}, \\
\dot{\mathbf{U}} & =\widehat{\mathbf{D}} \quad \text { for } \lambda_{1}=\lambda_{2}=\lambda_{3} \equiv \lambda, \\
\mathbf{K}_{\mathbf{U}} & \equiv \frac{2}{\mathrm{I}_{\mathbf{U}} \mathrm{II}_{\mathbf{U}}-\mathrm{III}_{\mathbf{U}}}\left\{\mathrm{I}_{\mathbf{U}} \mathrm{III}_{\mathbf{U}} \widehat{\mathbf{D}}+\mathrm{II}_{\mathbf{U}} \mathbf{U} \widehat{\mathbf{D}} \mathbf{U}-\mathrm{III}_{\mathbf{U}}(\mathbf{U} \widehat{\mathbf{D}}+\widehat{\mathbf{D}} \mathbf{U})\right\} .
\end{aligned}
$$

Substituting (5.2) and (5.5) into (4.12), the material time derivative of a general strain measure (5.1) is explicitly given as follows:

I. Distinct eigenvalues:

$$
\begin{gathered}
\dot{\mathbf{E}}=\mathbf{K}_{\mathbf{E}}+\sum_{i=1}^{3}\left\{\lambda_{i} f^{\prime}\left(\lambda_{i}\right) \operatorname{tr}\left(\widehat{\mathbf{D}} \mathbf{E}_{i}\right)-\operatorname{tr}\left(\mathbf{K}_{\mathbf{E}} \mathbf{E}_{i}\right)\right\} \mathbf{E}_{i}, \\
\mathbf{K}_{\mathbf{E}} \equiv \frac{2}{\mathrm{I}_{\mathbf{U}} \mathrm{II}_{\mathbf{U}}-\mathrm{III}_{\mathbf{U}}}\left\{a_{1} \mathrm{III}_{\mathbf{U}}\left(\mathrm{I}_{\mathbf{U}} \widehat{\mathbf{D}}-\mathbf{U} \widehat{\mathbf{D}}-\widehat{\mathbf{D}} \mathbf{U}\right)+a_{3} \mathbf{U} \widehat{\mathbf{D}} \mathbf{U}\right\} \\
\mathbf{E}_{i}=\frac{1}{\left(\lambda_{i}-\lambda_{j}\right)\left(\lambda_{i}-\lambda_{k}\right)}\left\{\mathbf{U}^{2}-\left(\lambda_{j}+\lambda_{k}\right) \mathbf{U}+\lambda_{j} \lambda_{k} \mathbf{1}\right\} \\
a_{1}=-\sum_{i=1}^{3} \frac{\left(\lambda_{j}+\lambda_{k}\right) f\left(\lambda_{i}\right)}{\left(\lambda_{i}-\lambda_{j}\right)\left(\lambda_{i}-\lambda_{k}\right)} \\
a_{3} \equiv a_{1} \mathrm{II}_{\mathbf{U}}+a_{2}\left(\mathrm{I}_{\mathbf{U}} \mathrm{II}_{\mathbf{U}}-\mathrm{III}_{\mathbf{U}}\right)=\sum_{i=1}^{3} \frac{\lambda_{i}^{2}\left(\lambda_{j}+\lambda_{k}\right) f\left(\lambda_{i}\right)}{\left(\lambda_{i}-\lambda_{j}\right)\left(\lambda_{i}-\lambda_{k}\right)}
\end{gathered}
$$

where $i, j, k$ are cyclic permutations of 1,2 , and 3 .

II. Repeated eigenvalues (i) $\lambda_{1} \neq \lambda_{2}=\lambda_{3}$ :

$$
\begin{gathered}
\dot{\mathbf{E}}=\mathbf{K}_{\mathbf{E}}+\left\{\lambda_{1} f^{\prime}\left(\lambda_{1}\right) \operatorname{tr}\left(\widehat{\mathbf{D}} \mathbf{E}_{1}\right)-\operatorname{tr}\left(\mathbf{K}_{\mathbf{E}} \mathbf{E}_{1}\right)\right\} \mathbf{E}_{1} \\
\mathbf{K}_{\mathbf{E}} \equiv \frac{2}{\mathrm{I}_{\mathbf{U}} \mathrm{II}_{\mathbf{U}}-\mathrm{III}_{\mathbf{U}}}\left\{a_{1} \mathrm{III}_{\mathbf{U}}\left(\mathrm{I}_{\mathbf{U}} \widehat{\mathbf{D}}-\mathbf{U} \widehat{\mathbf{D}}-\widehat{\mathbf{D}} \mathbf{U}\right)+a_{3} \mathbf{U} \widehat{\mathbf{D}} \mathbf{U}\right\} \\
\mathbf{E}_{1}=\frac{1}{\lambda_{1}-\lambda_{2}}\left(\mathbf{U}-\lambda_{2} \mathbf{1}\right) \\
a_{1}=-2 \lambda_{2} \frac{f\left(\lambda_{1}\right)-f\left(\lambda_{2}\right)}{\left(\lambda_{1}-\lambda_{2}\right)^{2}}+\frac{\lambda_{1}+\lambda_{2}}{\lambda_{1}-\lambda_{2}} f^{\prime}\left(\lambda_{2}\right) \\
a_{3}=2 \lambda_{1}^{2} \lambda_{2} \frac{f\left(\lambda_{1}\right)-f\left(\lambda_{2}\right)}{\left(\lambda_{1}-\lambda_{2}\right)^{2}}-\lambda_{2}^{2} \frac{\lambda_{1}+\lambda_{2}}{\lambda_{1}-\lambda_{2}} f^{\prime}\left(\lambda_{2}\right) .
\end{gathered}
$$


III. Repeated eigenvalues (ii) $\lambda_{1}=\lambda_{2}=\lambda_{3} \equiv \lambda$ :

$$
\dot{\mathbf{E}}=\lambda f^{\prime}(\lambda) \widehat{\mathbf{D}}
$$

It should be noted that (5.6b) can be obtained by taking limits as $\lambda_{3}$ goes to $\lambda_{2}$ in (5.6a). Similarly, (5.6c) can be obtained by taking limits as $\lambda_{2}$ goes to $\lambda_{1}=\lambda$ in (5.6b).

Concluding remarks. Many results, obtained in the past by various authors for isotropic tensor-valued functions of symmetric tensors, can be shown to remain valid even for nonsymmetric tensors when the eigenvalues are distinct. For symmetric tensors, repeated eigenvalues result in a lower-order polynomial tensor equation than provided by the Hamilton-Cayley theorem. This is not the case for general nonsymmetric tensors.

The material time derivative of any material strain measure of the general form (5.1) can be represented explicitly and in a coordinate-invariant form, in terms of the deformation rate tensor $\widehat{\mathbf{D}}$, and the stretch tensor $\mathbf{U}$ and its eigenvalues $\lambda_{i}$, without an eigenvector calculation; the eigenvalues of $\mathbf{U}$ are given explicitly in terms of its invariants, as discussed in Sec. 3.

Appendix A. Consider the equation

$$
\mathbf{A X}-\mathbf{X A}=\mathbf{B}
$$

where $\mathbf{A}$ and $\mathbf{B}$ are general nonsymmetric tensors such that

$$
\operatorname{tr}(\mathbf{B})=\operatorname{tr}(\mathbf{A B})=\operatorname{tr}\left(\mathbf{A}^{2} \mathbf{B}\right)=0 .
$$

Denote the eigenvalues of $\mathbf{A}$ by $\lambda_{i}, i=1,2,3$. First, consider the case where all three eigenvalues are distinct. In this case, the general solution $\mathbf{X}$ can be split into a part $\stackrel{*}{\mathbf{X}}$, coaxial with $\mathbf{A}$, and a (noncoaxial) remaining part $\mathbf{N}_{\mathbf{X}}$,

$$
\begin{gathered}
\mathbf{X}=\stackrel{*}{\mathbf{X}}+\mathbf{N}_{\mathbf{X}} \\
\stackrel{*}{\mathbf{X}}=\sum_{i=1}^{3}\left(\hat{\mathbf{e}}_{i} \cdot \mathbf{X} \mathbf{e}_{i}\right) \mathbf{e}_{i} \otimes \hat{\mathbf{e}}_{i} \\
\operatorname{tr}\left(\mathbf{N}_{\mathbf{X}}\right)=\operatorname{tr}\left(\mathbf{A} \mathbf{N}_{\mathbf{X}}\right)=\operatorname{tr}\left(\mathbf{A}^{2} \mathbf{N}_{\mathbf{X}}\right)=0
\end{gathered}
$$

where $\mathbf{e}_{i}$ and $\hat{\mathbf{e}}_{i}$ are the eigenvectors and reciprocal vectors of $\mathbf{A}$, corresponding to the eigenvalue $\lambda_{i}, i=1,2,3$. The coaxial part $\stackrel{*}{\mathbf{X}}$ has the form

$$
\stackrel{*}{\mathbf{X}}=\alpha_{0} \mathbf{1}+\alpha_{1} \mathbf{A}+\alpha_{2} \mathbf{A}^{2}
$$

where $\alpha_{0}, \alpha_{1}$, and $\alpha_{2}$ are constants. Now, consider only the noncoaxial part $\mathbf{N}_{\mathbf{X}}$ and seek to obtain a coordinate-invariant expression. Pre- and post-multiply (A.1) by $\mathbf{A}$ and subtract to obtain, in view of (A.3),

$$
\mathbf{A}^{2} \mathbf{N}_{\mathbf{X}}+\mathbf{N}_{\mathbf{X}} \mathbf{A}^{2}-2 \mathbf{A} \mathbf{N}_{\mathbf{X}} \mathbf{A}=\mathbf{A B}-\mathbf{B A}
$$


It follows from Rivlin's identities [10, Eqs. 4.22] and (A.3) that

$$
\mathbf{A} \mathbf{N}_{\mathbf{X}} \mathbf{A}=\mathrm{I}_{\mathbf{A}}\left(\mathbf{A} \mathbf{N}_{\mathbf{X}}+\mathbf{N}_{\mathbf{X}} \mathbf{A}\right)-\left(\mathbf{A}^{2} \mathbf{N}_{\mathbf{X}}+\mathbf{N}_{\mathbf{X}} \mathbf{A}^{2}\right)-\mathrm{II}_{\mathbf{A}} \mathbf{N}_{\mathbf{X}}
$$

Substitute (A.5) into (A.4) to arrive at

$$
\mathrm{CN}_{\mathbf{X}}+\mathbf{N}_{\mathbf{X}} \mathbf{C}=\mathbf{A B}-\mathbf{B A}
$$

where

$$
\mathbf{C} \equiv 3 \mathbf{A}^{2}-2 \mathrm{I}_{\mathbf{A}} \mathbf{A}+\mathrm{II}_{\mathbf{A}} \mathbf{1}
$$

Following Scheidler's [13] procedure, this can be solved for $\mathbf{X}$ as follows:

$$
\mathbf{X}=\frac{1}{\mathrm{III}_{\tilde{\mathbf{C}}}} \widetilde{\mathbf{C}}(\mathbf{A B}-\mathbf{B A}) \widetilde{\mathbf{C}}, \quad \widetilde{\mathbf{C}}=\mathrm{I}_{\mathbf{C}} \mathbf{1}-\mathbf{C},
$$

which can be rewritten as

$$
\begin{gathered}
\left(4 \mathrm{II}_{\mathbf{A}^{\prime}}^{3}+27 \mathrm{III}_{\mathbf{A}^{\prime}}^{2}\right) \mathbf{X}=4 \mathrm{II}_{\mathbf{A}^{\prime}}^{2}\left(\mathbf{A}^{\prime} \mathbf{B}-\mathbf{B A}^{\prime}\right)-9 \mathrm{III}_{\mathbf{A}^{\prime}}\left(\mathbf{A}^{\prime 2} \mathbf{B}-\mathbf{B} \mathbf{A}^{\prime 2}\right) \\
-3 \mathrm{II}_{\mathbf{A}^{\prime}} \mathbf{A}^{\prime}\left(\mathbf{A}^{\prime} \mathbf{B}-\mathbf{B A}^{\prime}\right) \mathbf{A}^{\prime} .
\end{gathered}
$$

The same result is obtained by Mehrabadi and Nemat-Nasser [9], and Guo et al. [3] assuming that $\mathbf{A}$ is symmetric and $\mathbf{X}$ is skewsymmetric. A similar result is obtained by Balendran and Nemat-Nasser [1] for the case where $\mathbf{A}$ is skewsymmetric and $\mathbf{X}$ is symmetric. Note that $\mathrm{III}_{\mathbf{A}}=0$ for skewsymmetric $\mathbf{A}$.

Now, consider the case where two of the three eigenvalues are equal, say $\lambda_{2}=\lambda_{3}$. Let the eigenvector and the reciprocal vector corresponding to $\lambda_{2}=\lambda_{3}$ be $\mathbf{e}_{2}$ and $\hat{\mathbf{e}}_{3}$, respectively. Then, $\mathbf{X}$ can be expressed as

$$
\begin{aligned}
& \mathbf{X}=\stackrel{*}{\mathbf{X}}+\mathbf{N}_{\mathbf{X}} \\
& \stackrel{*}{\mathbf{X}}=\left(\hat{\mathbf{e}}_{1} \cdot \mathbf{X} \mathbf{e}_{1}\right) \mathbf{e}_{1} \otimes \hat{\mathbf{e}}_{1}+\frac{1}{2}\left(\hat{\mathbf{e}}_{2} \cdot \mathbf{X} \mathbf{e}_{2}+\hat{\mathbf{e}}_{3} \cdot \mathbf{X} \mathbf{e}_{3}\right)\left(\mathbf{e}_{2} \otimes \hat{\mathbf{e}}_{2}+\mathbf{e}_{3} \otimes \hat{\mathbf{e}}_{3}\right) \\
& +\left(\hat{\mathbf{e}}_{2} \cdot \mathbf{X} \mathbf{e}_{3}\right) \mathbf{e}_{2} \otimes \hat{\mathbf{e}}_{3}, \\
& \mathbf{N}_{\mathbf{X}}=\mathbf{N}_{\overline{\mathbf{X}}}+\mathbf{Y}_{1}, \quad \mathbf{N}_{\overline{\mathbf{X}}}=\overline{\mathbf{A}} \mathbf{Y}_{2}-\mathbf{Y}_{2} \overline{\mathbf{A}}, \\
& \mathbf{Y}_{1}=\left(\hat{\mathbf{e}}_{2} \cdot \mathbf{X} \mathbf{e}_{2}-\hat{\mathbf{e}}_{3} \cdot \mathbf{X} \mathbf{e}_{3}\right)\left(\mathbf{e}_{2} \otimes \hat{\mathbf{e}}_{2}-\mathbf{e}_{3} \otimes \hat{\mathbf{e}}_{3}\right)+\left(\hat{\mathbf{e}}_{3} \cdot \mathbf{X} \mathbf{e}_{2}\right) \mathbf{e}_{3} \otimes \hat{\mathbf{e}}_{2}, \\
& \mathbf{Y}_{2}=\frac{1}{\lambda_{1}-\lambda_{2}} \sum_{j=2}^{3}\left\{\left(\hat{\mathbf{e}}_{1} \cdot \mathbf{X} \mathbf{e}_{j}\right) \mathbf{e}_{1} \otimes \mathbf{e}_{j}-\left(\hat{\mathbf{e}}_{j} \cdot \mathbf{X} \mathbf{e}_{1}\right) \mathbf{e}_{j} \otimes \mathbf{e}_{1}\right\},
\end{aligned}
$$

where

$$
\overline{\mathbf{A}}=\mathbf{A}-\mathbf{H}, \quad \mathbf{H}=\frac{1}{\lambda_{2}-\lambda_{1}}\left\{\mathbf{A}^{2}-\left(\lambda_{1}+\lambda_{2}\right) \mathbf{A}+\lambda_{1} \lambda_{2} \mathbf{1}\right\}
$$

Substitute (A.8) into (A.1) to obtain

$$
\begin{aligned}
\mathbf{Y}_{1}=\frac{1}{2 \mu_{23}} & \left(\hat{\mathbf{e}}_{2} \cdot \mathbf{B} \mathbf{e}_{2}-\hat{\mathbf{e}}_{33} \cdot \mathbf{B} \mathbf{e}_{3}\right) \mathbf{e}_{3} \otimes \hat{\mathbf{e}}_{2} \\
& -\frac{1}{2 \mu_{23}}\left(\hat{\mathbf{e}}_{3} \cdot \mathbf{B} \mathbf{e}_{2}\right)\left(\mathbf{e}_{2} \otimes \hat{\mathbf{e}}_{2}-\mathbf{e}_{3} \otimes \hat{\mathbf{e}}_{3}\right),
\end{aligned}
$$


where $\mu_{23}=\hat{\mathbf{e}}_{2} \cdot \mathbf{A e}_{3}$. Note that it is not trivial to obtain a coordinate-invariant expression for $\mathbf{Y}_{1}$. When $\mathbf{A}$ is symmetric,

$$
\mu_{23}=\hat{\mathbf{e}}_{2} \cdot \mathbf{B} \mathbf{e}_{2}=\hat{\mathbf{e}}_{3} \cdot \mathbf{B} \mathbf{e}_{3}=\hat{\mathbf{e}}_{3} \cdot \mathbf{B} \mathbf{e}_{2}=0 .
$$

Then $\mathbf{Y}_{1}$ is not unique. It follows from (A.9) that

$$
\overline{\mathbf{A}}^{2}-\left(\lambda_{1}+\lambda_{2}\right) \overline{\mathbf{A}}+\lambda_{1} \lambda_{2} \mathbf{1}=\mathbf{0}, \quad \overline{\mathbf{A}} \mathbf{H}=\mathbf{H} \overline{\mathbf{A}}=\lambda_{2} \mathbf{H}, \quad \mathbf{H}^{2}=\mathbf{0} .
$$

Hence from (A.8)

$$
\overline{\mathbf{A}} \mathbf{N}_{\overline{\mathbf{X}}}+\mathbf{N}_{\overline{\mathbf{X}}} \overline{\mathbf{A}}=\left(\lambda_{1}+\lambda_{2}\right) \mathbf{N}_{\overline{\mathbf{X}}}, \quad \mathbf{H} \mathbf{N}_{\overline{\mathbf{X}}} \mathbf{H}=\mathbf{0},
$$

and

$$
\begin{aligned}
\mathbf{H}\left(\overline{\mathbf{A}} \mathbf{N}_{\overline{\mathbf{X}}}\right. & \left.-\mathbf{N}_{\overline{\mathbf{X}}} \overline{\mathbf{A}}\right)-\left(\overline{\mathbf{A}} \mathbf{N}_{\overline{\mathbf{X}}}-\mathbf{N}_{\overline{\mathbf{X}}} \overline{\mathbf{A}}\right) \mathbf{H} \\
& =2\left(\mathbf{H} \overline{\mathbf{A}} \mathbf{N}_{\overline{\mathbf{X}}}+\mathbf{N}_{\overline{\mathbf{X}}} \overline{\mathbf{A}} \mathbf{H}\right)-\mathbf{H}\left(\overline{\mathbf{A}} \mathbf{N}_{\overline{\mathbf{X}}}+\mathbf{N}_{\overline{\mathbf{X}}} \overline{\mathbf{A}}\right)-\left(\overline{\mathbf{A}} \mathbf{N}_{\overline{\mathbf{X}}}+\mathbf{N}_{\overline{\mathbf{X}}} \overline{\mathbf{A}}\right) \mathbf{H} \\
& =\left(\lambda_{2}-\lambda_{1}\right)\left(\mathbf{H} \mathbf{N}_{\overline{\mathbf{X}}}+\mathbf{N}_{\overline{\mathbf{X}}} \mathbf{H}\right) .
\end{aligned}
$$

Pre- and post-multiply (A.1) by $\overline{\mathbf{A}}$, and subtract to obtain, in view of (A.8) and (A.12),

$$
\overline{\mathbf{A}}^{2} \mathbf{N}_{\overline{\mathbf{X}}}+\mathbf{N}_{\overline{\mathbf{X}}} \overline{\mathbf{A}}^{2}-2 \overline{\mathbf{A}} \mathbf{N}_{\overline{\mathbf{X}}} \overline{\mathbf{A}}=\overline{\mathbf{A}} \mathbf{B}-\mathbf{B} \overline{\mathbf{A}}+\left(\lambda_{1}-\lambda_{2}\right)\left(\mathbf{H} \mathbf{N}_{\overline{\mathbf{X}}}+\mathbf{N}_{\overline{\mathbf{X}}} \mathbf{H}\right)
$$

Substitute (A.11) into (A.5) to obtain

$$
\overline{\mathbf{A}} \mathbf{N}_{\overline{\mathbf{X}}} \overline{\mathbf{A}}=\lambda_{2}\left(\overline{\mathbf{A}} \mathbf{N}_{\overline{\mathbf{X}}}+\mathbf{N}_{\overline{\mathbf{X}}} \overline{\mathbf{A}}\right)-\lambda_{2}^{2} \mathbf{N}_{\overline{\mathbf{X}}}
$$

Substitute (A.14) into (A.13) to arrive at

$$
\begin{aligned}
\left(\lambda_{1}-\lambda_{2}\right)\left\{\overline{\mathbf{A}} \mathbf{N}_{\overline{\mathbf{X}}}+\mathbf{N}_{\overline{\mathbf{X}}} \overline{\mathbf{A}}-\right. & \left.2 \lambda_{2} \mathbf{N}_{\overline{\mathbf{X}}}\right\} \\
& =\overline{\mathbf{A}} \mathbf{B}-\mathbf{B} \overline{\mathbf{A}}+\left(\lambda_{1}-\lambda_{2}\right)\left(\mathbf{H} \mathbf{N}_{\overline{\mathbf{X}}}+\mathbf{N}_{\overline{\mathbf{X}}} \mathbf{H}\right) .
\end{aligned}
$$

Now, pre- and post-multiply (A.8d) by $\overline{\mathbf{A}}$, add together and substitute (A.11) to arrive at

$$
\overline{\mathbf{A}} \mathbf{N}_{\overline{\mathbf{X}}}+\mathbf{N}_{\overline{\mathbf{X}}} \overline{\mathbf{A}}=\left(\lambda_{1}+\lambda_{2}\right) \mathbf{N}_{\overline{\mathbf{X}}} .
$$

Substitution of (A.16) into (A.15) results in

$$
\mathbf{N}_{\overline{\mathbf{X}}}=\frac{1}{\left(\lambda_{1}-\lambda_{2}\right)^{2}}(\overline{\mathbf{A}} \mathbf{B}-\mathbf{B} \overline{\mathbf{A}})+\frac{1}{\left(\lambda_{1}-\lambda_{2}\right)}\left(\mathbf{H N}_{\overline{\mathbf{X}}}+\mathbf{N}_{\overline{\mathbf{X}}} \mathbf{H}\right)
$$

Hence, it follows from (A.11c) and (A.12b) that

$$
\mathbf{H N}_{\overline{\mathbf{X}}}+\mathbf{N}_{\overline{\mathbf{X}}} \mathbf{H}=\frac{1}{\left(\lambda_{1}-\lambda_{2}\right)^{2}}\{\mathbf{H}(\overline{\mathbf{A}} \mathbf{B}-\mathbf{B} \overline{\mathbf{A}})+(\overline{\mathbf{A}} \mathbf{B}-\mathbf{B} \overline{\mathbf{A}}) \mathbf{H}\} .
$$

Substitute (A.17b) into (A.17a) to arrive at

$$
\begin{aligned}
\mathbf{N}_{\overline{\mathbf{X}}}= & \frac{1}{\left(\lambda_{1}-\lambda_{2}\right)^{2}}(\overline{\mathbf{A}} \mathbf{B}-\mathbf{B} \overline{\mathbf{A}}) \\
& +\frac{1}{\left(\lambda_{1}-\lambda_{2}\right)^{3}}\{\mathbf{H}(\overline{\mathbf{A}} \mathbf{B}-\mathbf{B} \overline{\mathbf{A}})+(\overline{\mathbf{A}} \mathbf{B}-\mathbf{B} \overline{\mathbf{A}}) \mathbf{H}\} .
\end{aligned}
$$


Acknowledgment. The work reported here has been supported by the Army Research Office under Grant ARO-DAAL-03-92-G-0108 to the University of California, San Diego.

\section{REFERENCES}

[1] B. Balendran and S. Nemat-Nasser, Integration of inelastic constitutive equations for constant velocity gradient with large rotation, Appl. Math. Comput. 67, 161-195 (1995)

[2] D. E. Carlson and A. Hoger, The derivative of a tensor-valued function of a tensor, Quart. Appl. Math. XLIV, no. 3, 409-423 (1986)

[3] Z.-h. Guo, Th. Lehmann, H. Liang, and C.-s. Man, Twirl tensors and the tensor equation AX $\mathbf{X A}=\mathbf{C}$, J. Elasticity 27, 227-245 (1992)

[4] M. E. Gurtin and K. Spear, On the relationship between the logarithmic strain rate and the stretching tensor, Internat. J. Solids and Structures 19, no. 5, 437-444 (1983)

[5] R. Hill, On constitutive inequalities for simple materials. I, J. Mech. Phys. Solids 16, 229 (1968)

[6] R. Hill, Constitutive inequalities for isotropic elastic solids under finite strain, Proc. Roy. Soc. London Ser. A 314, 457-472 (1970)

[7] R. Hill, Aspects of invariance in solid mechanics, Adv. in Appl. Mech. 18, 1-75 (1978)

[8] A. Hoger, The material time derivative of logarithmic strain, Internat. J. Solids and Structures 22, no. 9, 1019-1032 (1986)

[9] M. M. Mehrabadi and S. Nemat-Nasser, Some basic kinematical relations for finite deformations of continua, Mech. Materials 6, 127-138 (1987)

[10] R. S. Rivlin, Further remarks on the stress-deformation relations for isotropic materials, J. Rational Mech. Anal. 4, 681-701 (1955)

[11] M. Scheidler, Time rates of generalized strain tensors, Part I: Component formulas, Mech. Materials 11, 199-210 (1991)

[12] M. Scheidler, Time rates of generalized strain tensors, Part II: Approximate basis-free formulas, Mech. Materials 11, 211-219 (1991)

[13] M. Scheidler, The tensor equation $\mathbf{A X}+\mathbf{X A}=\Phi(\mathbf{A}, \mathbf{H})$, with applications to kinematics of continua, J. Elasticity 36, 117-153 (1994)

[14] T. C. T. Ting, Determination of $\mathbf{C}^{1 / 2}, \mathbf{C}^{-1 / 2}$ and more general isotropic tensor functions of $\mathbf{C}$, J. Elasticity 15, 319-323 (1985) 